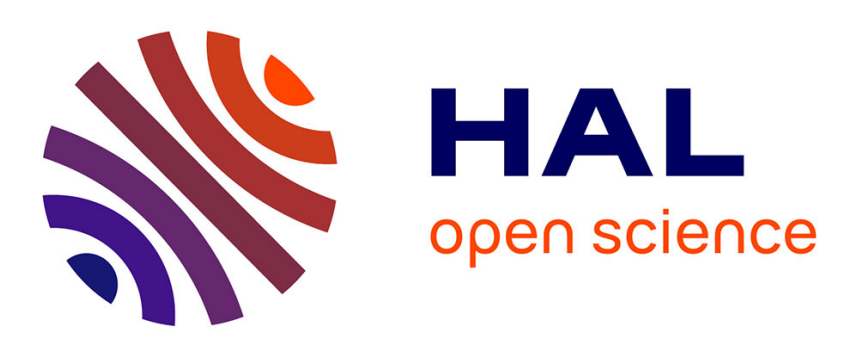

\title{
Lyman- $\alpha$ radiation of a probing metastable hydrogen beam to measure electric fields in diluted fluids and plasmas
}

\author{
Aurelien Lejeune, Fabrice Doveil, Laurence Chérigier-Kovacic
}

\section{To cite this version:}

Aurelien Lejeune, Fabrice Doveil, Laurence Chérigier-Kovacic. Lyman- $\alpha$ radiation of a probing metastable hydrogen beam to measure electric fields in diluted fluids and plasmas. Physics of Plasmas, 2013, 20, pp.055701. 10.1063/1.4801510 . hal-03534310

\author{
HAL Id: hal-03534310 \\ https://hal.science/hal-03534310
}

Submitted on 19 Jan 2022

HAL is a multi-disciplinary open access archive for the deposit and dissemination of scientific research documents, whether they are published or not. The documents may come from teaching and research institutions in France or abroad, or from public or private research centers.
L'archive ouverte pluridisciplinaire HAL, est destinée au dépôt et à la diffusion de documents scientifiques de niveau recherche, publiés ou non, émanant des établissements d'enseignement et de recherche français ou étrangers, des laboratoires publics ou privés. 


\section{AIP Plasmas}

\section{Lyman- $\alpha$ radiation of a probing metastable hydrogen beam to measure electric fields in diluted fluids and plasmas}

F. Doveil, A. Lejeune, and L. Chérigier-Kovacic

Citation: Phys. Plasmas 20, 055701 (2013); doi: 10.1063/1.4801510

View online: http://dx.doi.org/10.1063/1.4801510

View Table of Contents: http://pop.aip.org/resource/1/PHPAEN/v20/i5

Published by the American Institute of Physics.

\section{Additional information on Phys. Plasmas}

Journal Homepage: http://pop.aip.org/

Journal Information: http://pop.aip.org/about/about_the_journal

Top downloads: http://pop.aip.org/features/most_downloaded

Information for Authors: http://pop.aip.org/authors

\section{ADVERTISEMENT}

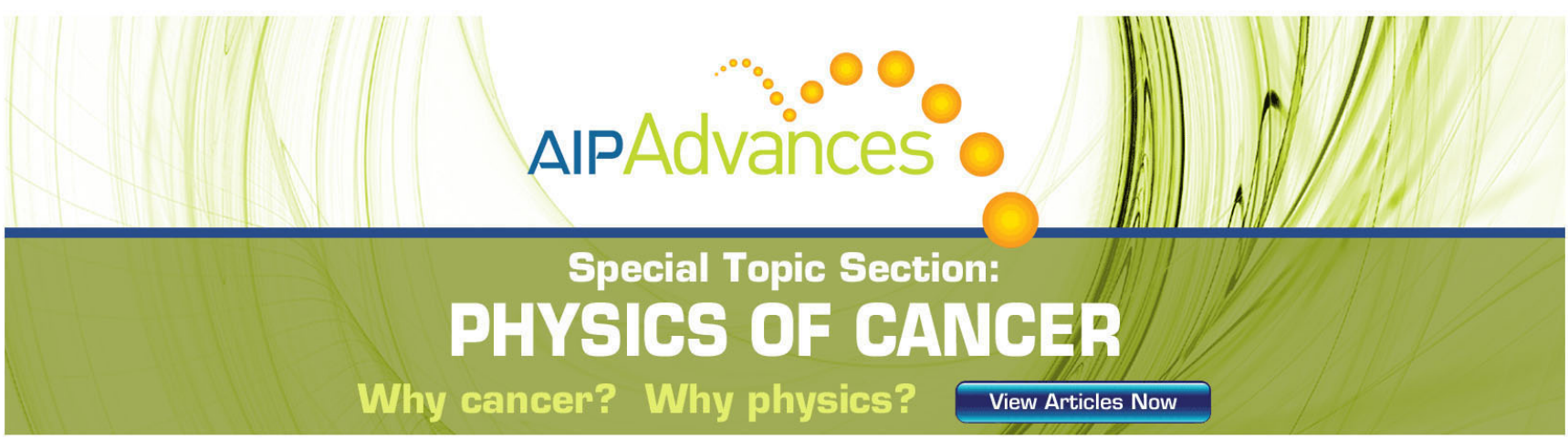




\title{
Lyman- $\alpha$ radiation of a probing metastable hydrogen beam to measure electric fields in diluted fluids and plasmas ${ }^{a)}$
}

\author{
F. Doveil, ${ }^{b), c)}$ A. Lejeune, ${ }^{\text {d),e) }}$ and L. Chérigier-Kovacic ${ }^{\mathrm{f})}$ \\ Aix Marseille Université, CNRS, PIIM UMR 7345, FR-13397 Marseilles Cedex 20, France
}

(Received 26 October 2012; accepted 12 December 2012; published online 15 April 2013)

\begin{abstract}
The interaction between a metastable $\mathrm{H}(2 s)$ atomic hydrogen beam and an external electric field leads to the emission of the Lyman- $\alpha$ line. It originates in the Stark mixing of the neardegenerate $2 s_{1 / 2}$ and $2 p_{1 / 2}$ levels separated by the Lamb shift. The quenched radiation proportional to the square of the electric field amplitude is recovered in vacuum by using such an atomic probe beam. For larger electric field, saturation is observed and related to the beam finite transit time. We also observe the strong enhancement of the signal when the field is oscillating at the Lamb shift frequency. This technique is applied in a plasma, offering an alternative way to measure weak electric fields by direct and non-intrusive means. (C) 2013 AIP Publishing LLC. [http://dx.doi.org/10.1063/1.4801510]
\end{abstract}

\section{INTRODUCTION}

Measuring electric fields with high space-time resolution and sensitivity has always been a critical problem in experimental classical physics, specially in diluted fluids and plasmas. For example, direct but intrusive double probe measurements can be used aboard satellites with good accuracy ${ }^{1}$ but cannot be easily used in laboratory plasmas where the probes strongly perturb the plasma. ${ }^{2}$ Heavy ion beams have been successfully used in fusion plasmas but only work in this particular environment and lead to indirect measurements. ${ }^{3,4}$ Laser Induced Fluorescence has been extensively used in laboratory plasmas, ${ }^{5}$ and specially ion thrusters, ${ }^{6}$ but the electric field is again indirectly deduced from the ion distribution function measurements.

In this paper, we show that the strong interaction between a constant or oscillatory electric field and an atomic hydrogen beam can be exploited ${ }^{7}$ to obtain sensitive measurements of electric fields with high space-time resolution and exceptional sensitivity. ${ }^{8}$ As is well known, for the hydrogen-like family (H, D, T, HeII, LiIII, BeIV, CVI), the atomic system possesses a first excited state which contains two nearly degenerate levels $\left(2 s_{1 / 2}, 2 p_{1 / 2}\right)$ with very different lifetimes, but separated in energy by a small amount, the famous Lamb shift. ${ }^{9}$ As a consequence, the ratio of lifetime differences to energy difference is unusually large in such an atomic system. Mixing of these levels by weak electric fields (as low as $1 \mathrm{~V} / \mathrm{cm}$ or less) largely enhances the intensity of the characteristic Lyman- $\alpha$ radiation leading to an effect which is several orders of magnitude more sensitive than other Stark processes.

We describe a technique based on (i) the injection of a metastable hydrogen atoms probe beam into the diagnosed

\footnotetext{
a)Paper GI3 1, Bull. Am. Phys. Soc. 57, 109 (2012).

${ }^{\mathrm{b})}$ Invited speaker.

c) fabrice.doveil@univ-amu.fr.

${ }^{d)}$ Present address: CAMECA, 92230 Gennevilliers, France.

e)aurelien.lejeune@ametek.com.

${ }^{\mathrm{f})}$ laurence.kovacic@univ-amu.fr.
}

volume,${ }^{10}$ and (ii) the measurement of the enhanced Lyman- $\alpha$ emission, which is accurately determined by the field. A proton beam is first extracted from a multipolar hydrogen plasma source. The atomic beam is then created either by collisions with $\mathrm{H}_{2}$ residual gas along the beam path or by resonant charge exchange in a cell containing Cesium vapor. In this latter case, the beam energy is chosen to maximize this charge exchange process.

This paper is organized as follows: In Sec. II, we recall the minimum theoretical background necessary to understand the quadratic law for the intensity of the Lyman- $\alpha$ radiation as a function of the electric field amplitude and to show the role played in our measurements by the Lamb shift. Section III describes the experiment. In Sec. IV A, we show experimental data in vacuum recovering the quadratic law and subsequent saturation at large field obtained at first from an ion beam, and in section IV B from an atomic $\mathrm{H}(2 s)$ beam sent between two polarized plates creating a dc-electric field between 0 and $100 \mathrm{~V} / \mathrm{cm}$ in vacuum. By a proper calibration, the intensity of the probing beam Lyman- $\alpha$ radiation can thus be used to obtain a local measurement of the electric field. The results in Sec. IV C furthermore exhibit the huge enhancement of the signal obtained when polarizing the plates with a RF voltage resonant with the Lamb frequency. Section $\mathrm{V}$ deals with the first application of this new diagnostic to the measurement of the Debye sheath in a plasma. And finally, Sec. VI gives our conclusion.

\section{THEORETICAL BACKGROUND}

In the Dirac theory describing the fine structure of hydrogen and hydrogenic atoms, the levels $2 s_{1 / 2}$ and $2 p_{1 / 2}$, which have the same value of total angular momentum quantum number but different values of orbital angular momentum, are actually said to be near-degenerate since it has been experimentally shown by Lamb and Retherford in 1947 that, contrary to the predictions of the Dirac theory, the $2 s$ state does not have exactly the same energy as the $2 p$ state. This last state lies higher by a small amount corresponding to a 
frequency $\nu_{0}=1057 \mathrm{MHz}$, the well-known Lamb shift extensively studied since then, both experimentally to precisely measure the shift ${ }^{11}$ and theoretically to understand its origins. ${ }^{12}$ Radiative corrections, due to interactions between the electron and electromagnetic fluctuations of vacuum, are responsible for these energy shifts called Lamb-shifts which are very important for our experiment when an electric field resonant with the Lamb frequency is applied to the atomic beam as will be shown later.

As in the Lamb experiment, we take advantage of the fact that the $2 s_{1 / 2}$ level is metastable (lifetime of about $0.14 \mathrm{~s}$ ), the transition to the ground state $1 s_{1 / 2}$ being forbidden by a selection rule $\Delta \ell= \pm 1$ for the orbital angular momentum. However, a transition can occur if the atoms are sent through a region where an electric field is applied. The quenching of the metastable $2 s$ state of hydrogen and hydrogenic atoms in the presence of externally applied electric fields leads to the production of a Lyman- $\alpha$ radiation with an intensity:

$$
I_{\alpha}=\Omega \mathcal{V} n_{2 s} \gamma_{2 s}^{*}
$$

where $\Omega$ is a geometric factor, $\mathcal{V}$ is the interacting volume, $n_{2 \mathrm{~s}}$ is the $2 s$ metastable hydrogen atom density, and $\gamma_{2 s}^{*}=$ $1 / \tau_{2 \mathrm{~s}}^{*}$ is the quenched $2 s$ transition rate related to the reduced lifetime $\tau_{2 \mathrm{~s}}^{*}$. It is known to result from Stark mixing between the $2 s$ and $2 p$ levels. As long as the magnitude of the applied electric field is large enough for the fine structure to be unimportant, the Stark effect can be treated like a perturbation of the non relativistic Hamiltonian $H_{0}$ of the unperturbed hydrogen atom where the $n=2$ level is four times degenerate. This is true for very high electric fields like those involved in lasers. The Stark splitting is then linear and partially removes this degeneracy. Lower fields have to be considered as the perturbation of the nearly degenerate system of levels $2 s_{1 / 2}$ and $2 p_{1 / 2}$, especially below the limit of $475 \mathrm{~V} / \mathrm{cm}$, when the Stark shift becomes negligible compared to the Lamb-shift. Bethe and Salpeter ${ }^{13}$ have derived equations for the populations of two levels having the same value of quantum numbers $n$ and $j$ in low electric fields, but not necessarily lower than this limit. We follow here ${ }^{12}$ in order to calculate the decay rate $\gamma_{2 s}^{*}$ of the $2 s_{1 / 2}$ level to the ground level (details of calculation can be found in more recent works like Ref. 14 or 15). The time-dependent Schrödinger equation is solved using the perturbation theory when an oscillating electric field of magnitude $E_{0}$ lower than $475 \mathrm{~V} / \mathrm{cm}$ and frequency $\omega / 2 \pi$ is applied to the nearly degenerate system of levels $2 s_{1 / 2}$ and $2 p_{1 / 2}$. Neglecting the terms in which the small $\gamma_{2 s}$ decay rate appears, the time-dependent equations for these levels are

$$
\begin{gathered}
i \hbar \frac{\partial}{\partial t} c_{2 s}(t)=V(t) e^{i \omega_{0} t} c_{2 p}(t), \\
i \hbar \frac{\partial}{\partial t} c_{2 p}(t)=V^{*}(t) e^{-i \omega_{0} t} c_{2 s}(t)-i \hbar \frac{\gamma_{2 p}}{2} c_{2 p}(t),
\end{gathered}
$$

where $c_{2 \mathrm{~s}}(t)$ and $c_{2 \mathrm{p}}(t)$ are the probability amplitudes, $\gamma_{2 \mathrm{p}}$ $=1 / \tau_{2 \mathrm{p}}$ is the decay rate of the $2 p$ state and $\hbar \omega_{0}$ is the energy difference associated to the Lamb-shift $\left(\nu_{0}=1057 \mathrm{MHz}\right)$. With $e$ the elementary charge and $a_{0}$ the Bohr radius, $V(t)$ $=\left\langle 2 s_{1 / 2}|e \mathbf{r} \cdot \mathbf{E}(t)| 2 p_{1 / 2}\right\rangle \propto e a_{0}\left|E_{0}\right| \exp (-i \omega t)$ is the matrix element of the perturbing Hamiltonian coupling $2 s_{1 / 2}$ and $2 p_{1 / 2}$. The exact solution $c_{2 s}(t)$ is the sum of two exponential terms. One of them being highly damped, it can be neglected and we assume $c_{2 s}(t)=e^{-\mu t}$. The system of Eqs. (2) can be solved analytically making the assumption $\mu \ll \gamma_{2 p} / 2$, leading to a field magnitude lower than $200 \mathrm{~V} / \mathrm{cm}$. Considering only the secular term, the effective decay rate is then given by

$$
\gamma_{2 s}^{*}(\omega)=\mu+\mu^{*}=\frac{9 e^{2} a_{0}^{2} E_{0}^{2}}{\hbar^{2}} \frac{\gamma_{2 p}}{\left(\omega-\omega_{0}\right)^{2}+\gamma_{2 p}^{2} / 4} .
$$

In the limit of a constant field when $\omega \rightarrow 0$, we also find the result of Lamb in Ref. 12

$$
\gamma_{2 s}^{*}(0)=\frac{9 e^{2} a_{0}^{2} E_{0}^{2}}{\hbar^{2}} \frac{\gamma_{2 p}}{\omega_{0}^{2}+\gamma_{2 p}^{2} / 4} .
$$

Obviously, the lifetime $\tau_{2 s}^{*}=1 / \gamma_{2 s}^{*}$ of $2 s_{1 / 2}$ in the presence of electric field remains greater than the natural lifetime $\tau_{2 p}=1.6 \times 10^{-9} \mathrm{~s}$ of $2 p_{1 / 2}$ and smaller than the natural lifetime $(0.14 \mathrm{~s})$ of $2 s_{1 / 2}$. For larger fields, the lifetime reduces to $2 \tau_{2 p} .{ }^{13}$ Calculating the quenched level $2 s$ lifetime is a major issue since the probe $\mathrm{H}(2 s)$ beam starts to interact with the perturbing field as soon as it is sent through the explored medium. One has to be sure that the probe beam is not totally quenched before it reaches the region of interest. As an example, according to Eqs. (3) and (4), Fig. 1 shows the lifetime of $2 s_{1 / 2}$ (solid line, left scale) as a function of the frequency at a field of magnitude $E_{0}=0.1 \mathrm{~V} / \mathrm{cm}$. The resonance amplification $\gamma_{2 s}^{*}(\omega) / \gamma_{2 s}^{*}(0)$ is also displayed in Fig. 1 (dashed line, right scale), featuring the strong enhancement in Eq. (3) when $\omega$ approaches $\omega_{0}$. At the resonance, we thus expect an amplification factor of about 440 , which is independent of the value of the field. This accounts for the theoretical ability to detect small constant fields as low as $1 \mathrm{~V} / \mathrm{cm}$ and even lower when the field frequency approaches the

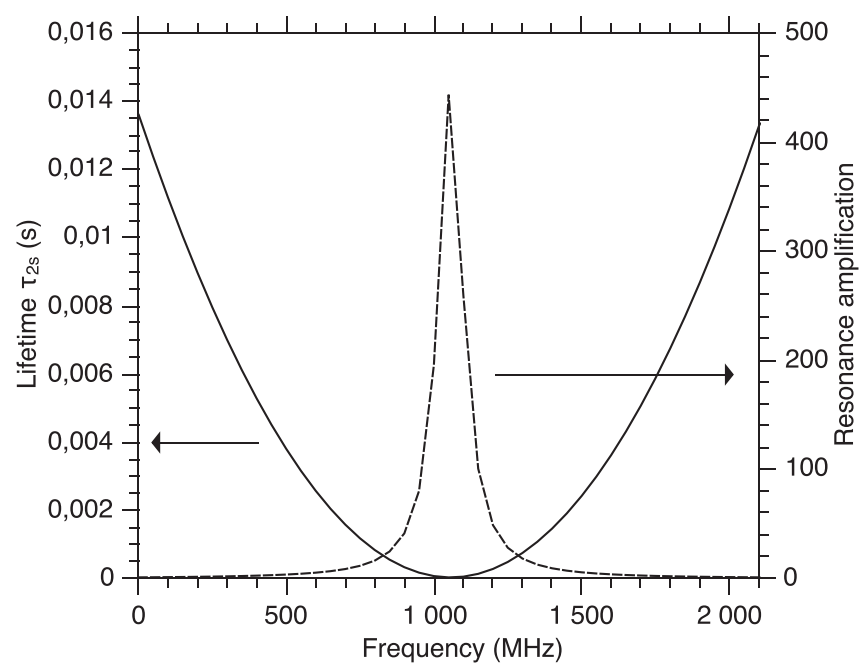

FIG. 1. Lifetime of $2 s_{1 / 2}$ level (solid line, left scale, $\left|E_{0}\right|=10 \mathrm{~V} / \mathrm{m}$ ) and resonance amplification factor $\gamma_{2 s}^{*}(\nu) / \gamma_{2 s}^{*}(0)$ (dashed line, right scale) versus frequency of the perturbing field. 
Lamb-shift frequency. The actual experimental resolution depends on the detection system. As we shall see in the following, we experimentally observe a factor of this order of magnitude between the signal measured when the electric field is resonant with the Lamb shift frequency and when the electric field is constant.

\section{EXPERIMENTAL SET-UP}

A view of the experimental setup ${ }^{16}$ is displayed in Fig. 2. The source of ions ${ }^{17}$ is a hydrogen plasma produced by a hot cathode discharge and confined by a magnetic multipole (number (1) in Fig. 2). A W-shaped tungsten filament (cathode) is heated by a current up to $15 \mathrm{~A}$ and is negatively biased (typical discharge voltage $V_{d}=-80 \mathrm{~V}$ ) with respect to the wall (anode). A discharge current up to $3 \mathrm{~A}$ is established, the primary electrons being responsible for the dissociation and ionization of $\mathrm{H}_{2}$, introduced in the chamber at a pressure ranging from about $10^{-5}$ to $10^{-4} \mathrm{mbar}$. The plasma contains different charged species such as $\mathrm{H}^{+}, \mathrm{H}_{2}^{+}$, and $\mathrm{H}_{3}^{+}$, which population ratio can be measured by a custom mass spectrometer in the measurement chamber (number (3) in Fig. 2). The ion extraction focusing Einzel lens is a conventional three stainless steel electrodes separated by ceramics. The first electrode is biased to a positive potential and the second is biased to a very negative potential, in order to decelerate the electrons and focus the beam. The potential difference between the third electrode which is simply grounded and the wall of the ion source can be varied between 0 and $500 \mathrm{~V}$ and sets the kinetic energy of the extracted ion beam. A temperature-monitored charge exchange cell containing cesium (Cs) vapor (number (2) in Fig. 2), built from a previously existing device, ${ }^{18}$ is used to produce the metastable hydrogen atoms from the proton in the ion beam according to the charge-exchange reaction $\mathrm{H}^{+}+\mathrm{Cs} \rightarrow \mathrm{H}(2 \mathrm{~s})+\mathrm{Cs}^{+}$. We work with a $500 \mathrm{eV}$ ion beam, which corresponds to the maximum cross section of this charge exchange process. ${ }^{19}$ The resulting atomic beam is then injected into a measurement chamber (number (3) in Fig. 2) where two horizontal parallel plates separated by $5 \mathrm{~cm}$ can be externally biased to create a static or oscillating electric field. One plate is grounded, while the other can be biased. This system of two plates with fixed separation can also be moved vertically. The Lyman- $\alpha$ light $(\lambda=121.6 \mathrm{~nm})$ is collected in a direction perpendicular to the beam by a lithium fluoride (LiF) lens and detected by a

(3)

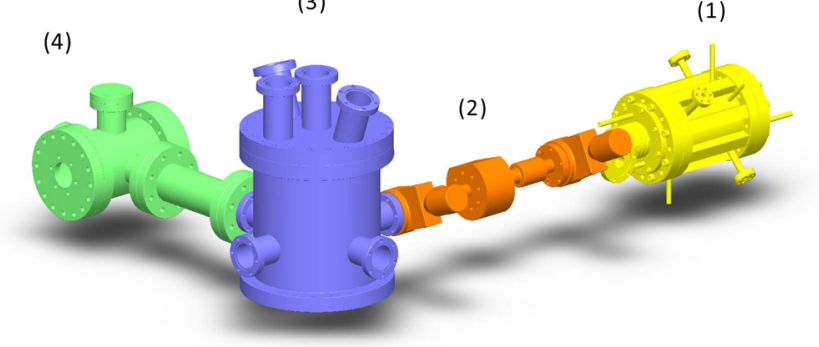

FIG. 2. Schematic view of the apparatus: ${ }^{1}$ Hydrogen plasma source with ion beam extraction lenses, ${ }^{2}$ Cs vapor charge exchange chamber, ${ }^{3}$ interaction chamber, ${ }^{4}$ Lyman- $\alpha$ detection chamber.
VUV-photomultiplier operating in vacuum (number (4) in Fig. 2). Spatial resolution is only determined by the size of the photocathode and the lens magnification. In order to improve the signal to noise ratio, a lock-in amplifier is used to detect the pulsed emission, for either a modulated ion beam extraction with a very low frequency (typically $10 \mathrm{~Hz}$ ) or for a modulated biasing of the non-grounded plate at a low frequency (typically $1 \mathrm{kHz}$ ). In this latter case, the continuous background of spontaneous emission from the beam is not recorded, only the part of the signal due to the field, synchronized with the biased plate modulation, is observed. The hydrogen pressure in the measurement chamber is usually about one third of the pressure in the source. This allows us to make measurements even with a pure ion beam through collisions between ions and the residual gas. This pressure can also be varied with an independent Argon gas introduction and a DC discharge can be created from a heated filament in the measurement chamber.

\section{ELECTRIC FIELD IN VACUUM}

\section{A. Ion beam probe}

Figure 3 features the intensity of the Lyman- $\alpha$ radiation obtained when there is no Cs in the path of the ion beam, as a function of the perturbing voltage applied between the plates for a source pressure of $1.7 \cdot 10^{-5}$ mbar and a discharge current of $2.5 \mathrm{~A}$. The bias voltage is pulsed at a frequency of $1 \mathrm{kHz}$. For each bias voltage, we give the minimum (circles) and maximum (squares) measured signal as an indication of the error bars. As predicted by the theory, we observe an E-square law as shown by the parabolic continuous curve for applied potential lower than $120 \mathrm{~V}$ (electric field strength lower than $24 \mathrm{~V} / \mathrm{cm}$ since the plates distance is $5 \mathrm{~cm}$ ). For larger electric field saturation occurs and can be explained considering the reduced lifetime of the emitting particles, $\tau_{2 s}^{*} \propto 1 / E_{0}^{2}$ being shorter and shorter when $E_{0}$ increases, whereas their transit time inside the observed region remains constant.

Since such a signal was not expected from the ion beam, we tried to vary the ion ratio in the beam to determine the

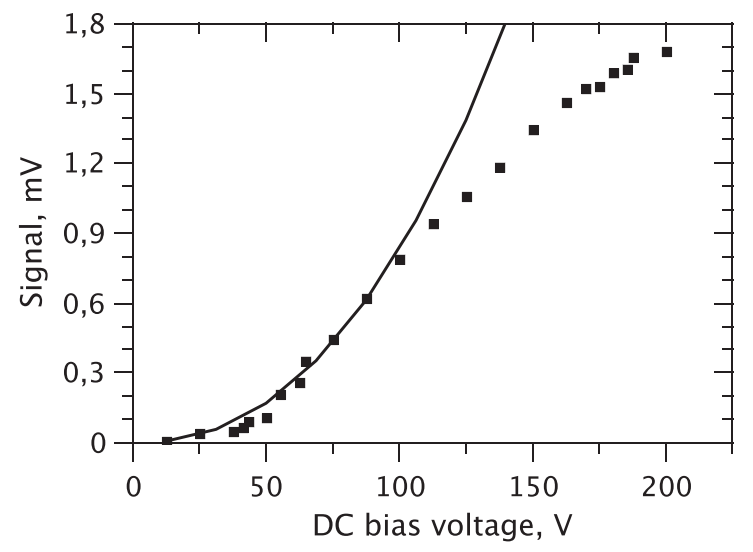

FIG. 3. Lyman- $\alpha$ emission versus static potential applied between the plates, for the probing ion beam without conversion in Cs cell; solid line is a parabola fitted to the data; source pressure $P=1.7 \cdot 10^{-5}$ mbar and discharge current $I_{d}=2.5 \mathrm{~A}$. 
source of this emission. Figure 4(a) shows the behaviour of the measured Lyman- $\alpha$ radiation intensity (minimum, maximum, and mean) as a function of the plasma source hydrogen pressure for a DC bias of $50 \mathrm{~V}$ between the plates. It also shows the decrease of the total ion beam current with pressure. We observe a monotonic increase of the Lyman- $\alpha$ signal with a slope that strongly diminishes for a pressure higher than $5 \cdot 10^{-5}$ mbar. Figure $4(\mathrm{~b})$ shows the relative density of $\mathrm{H}^{+}, \mathrm{H}_{2}^{+}$, and $\mathrm{H}_{3}^{+}$ions inside the beam measured with the ion mass spectrometer and predicted by a specific ion population evolution model inside the plasma source as a function of the plasma source hydrogen pressure. One may at first glance relate the Lyman- $\alpha$ intensity evolution to the production of $\mathrm{H}_{3}^{+}$, but the theory described above only applies to a special energy levels scheme with near degenerate levels, which can hardly be found in $\mathrm{H}_{3}^{+}$. The intensity of the emission being proportional to the gas pressure in the source, we may conclude that this radiation is emitted by $\mathrm{H}(2 s)$ atoms resulting from the reaction between $\mathrm{H}^{+}$or $\mathrm{H}_{2}^{+}$ ions and $\mathrm{H}_{2}$ molecules of the residual gas along the path of the beam in the measurement chamber. This signal is also proportional to the beam intensity which is observed to decrease above $5 \cdot 10^{-5}$ mbar.
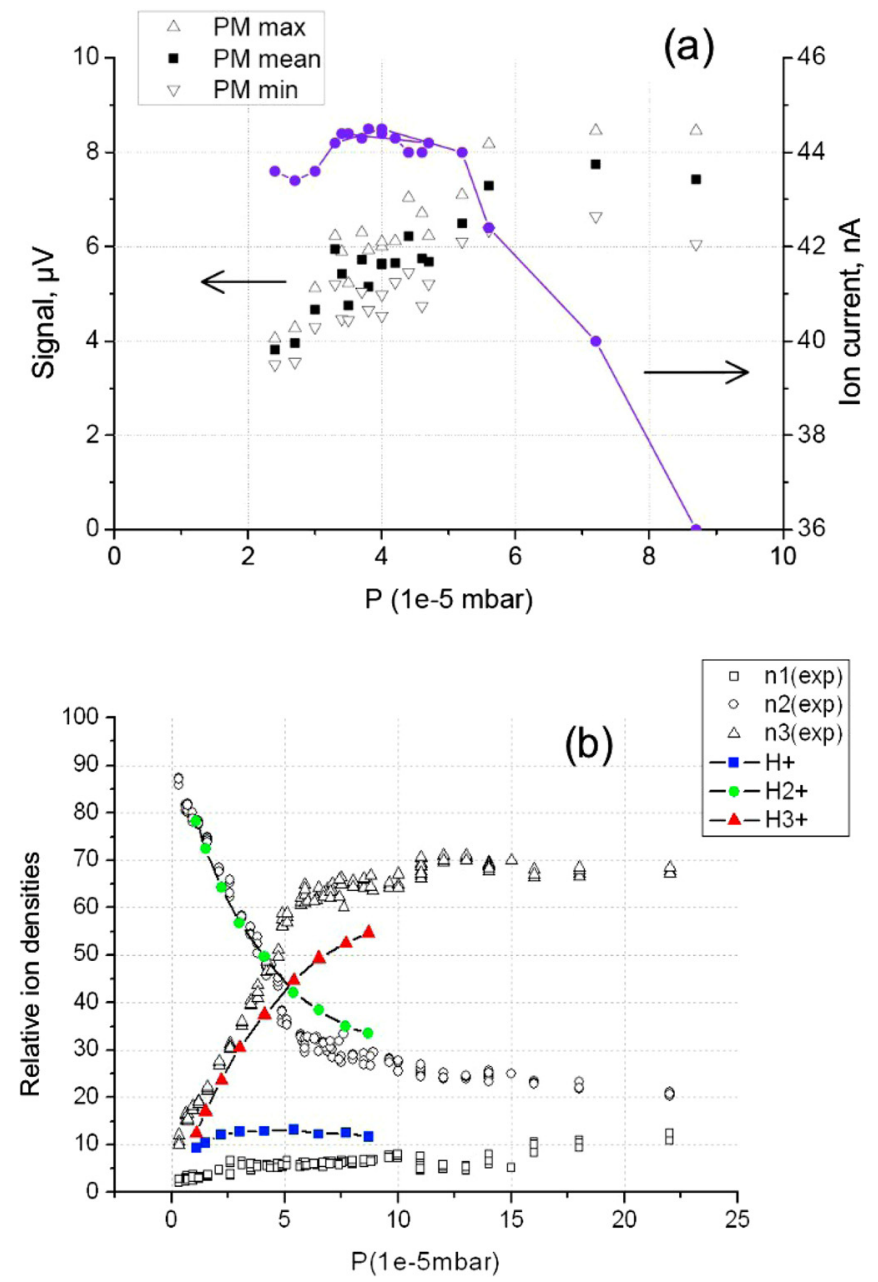

FIG. 4. (a) Lyman- $\alpha$ emission and ion beam current versus pressure for a static potential applied between the plates of $50 \mathrm{~V}$; (b) mass spectrometer measurement of the ion beam composition, continuous curves result from a global model of populations in the source plasma.

\section{B. Atomic beam probe}

The plot in Fig. 5 represents the intensity of the Lyman- $\alpha$ radiation as a function of the perturbing voltage (again modulated at $1 \mathrm{kHz}$ ) applied between the plates in the case of an atomic beam $\mathrm{H}(2 s)$ prepared in the Cs cell as previously described, for the same discharge conditions as in the previous case. As predicted by Eq. (3), the variation law is quadratic for applied potential lower than $120 \mathrm{~V}$ (electric field lower than $24 \mathrm{~V} / \mathrm{cm}$ ). As expected, the signal is much higher, by a factor of 4.5, than the signal obtained without Cs. As shown by mass spectrometer measurements, the $\mathrm{H}^{+}$ions represent about $10 \%$ of the total ion beam population, the $\mathrm{H}^{+}$production in the source plasma staying almost constant upon the working pressure range $10^{-4}-10^{-5} \mathrm{mbar}$ and the observed Lyman- $\alpha$ signal is not very dependent on the pressure either. As in the previews results presented in Fig. 3 for the ion beam probe, we observe for the atomic beam probe a saturation of the signal for applied potentials above $200 \mathrm{~V}$ (electric fields above $40 \mathrm{~V} / \mathrm{cm}$ ), which originates in the finite transit time of the emitters between the plates and in the diagnosed volume. $^{20}$

\section{RF resonance and Lamb shift}

In order to prove the great capacity of this new method to measure weak electric fields, we now verify the enhancement predicted by the calculation when the frequency of the applied electric field is resonant with the Lamb-shift frequency (Eq. (3) with $\omega \rightarrow \omega_{0}$ ). We produce a radiofrequency field in our measurement chamber using the lower plate as an emitting antenna. In this case, the lock-in amplifier is synchronized with a $1 \mathrm{kHz}$ reference modulation frequency. The transmission and reflection spectra in the chamber are controlled via a network analyzer connected to a probe located nearby the upper plate.

Figure 6 shows the intensity of the Lyman- $\alpha$ radiation as a function of the applied electric field frequency with constant amplitude. It is remarkable that the observed spectrum strongly recalls the hyperfine structure of $\mathrm{H}(2 s)$ hydrogen atoms. $^{21}$

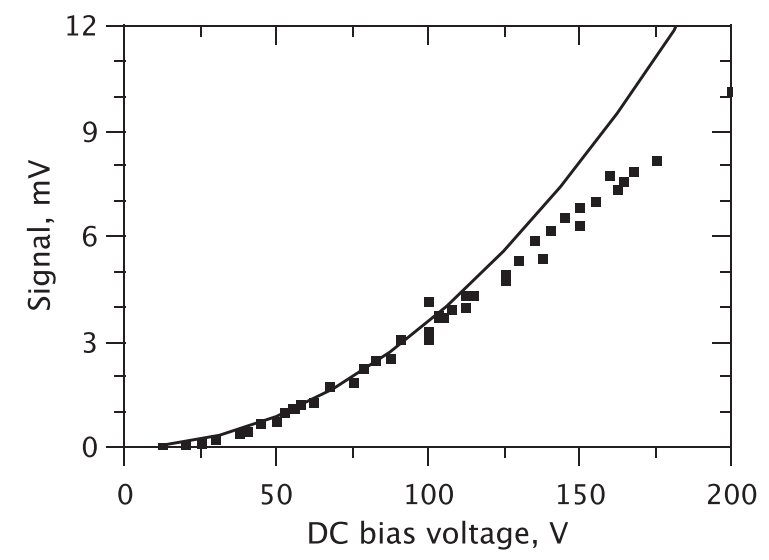

FIG. 5. Lyman- $\alpha$ emission versus static potential applied between the plates for the probing atomic beam; solid line is a parabola fitted to the data; source pressure $P=1.7 \cdot 10^{-5} \mathrm{mbar}$ and discharge current $I_{d}=2.5 \mathrm{~A}$. 


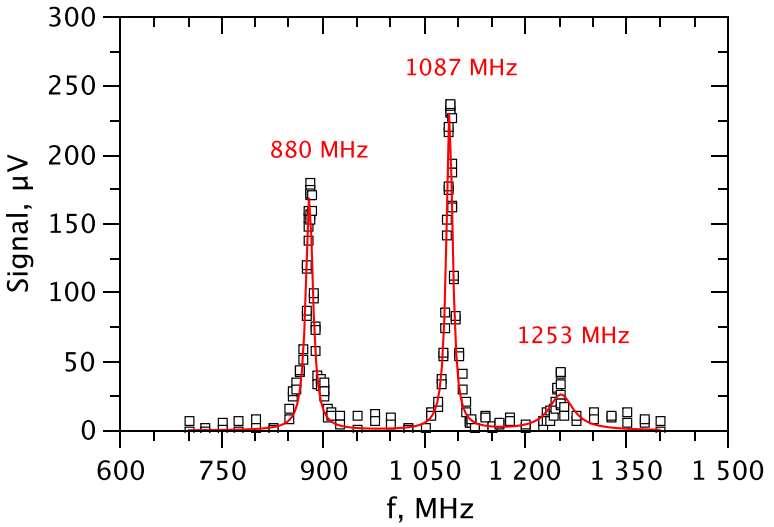

FIG. 6. Spectrum of $\mathrm{H}(2 s)$ Lyman- $\alpha$ radiation measured for a fixed RF amplitude of $1 \mathrm{~V}$; source pressure $P=2.7 \cdot 10^{-5} \mathrm{mbar}$ and discharge current $I_{d}=2.0 \mathrm{~A}$

Figure 7 again displays the quadratic law for an oscillating field at the Lamb-shift frequency (corresponding to the maximum peak at $1087 \mathrm{MHz}$ in the previous figure) followed by a strong saturation when the RF voltage amplitude is increased. The parabolic fit clearly shows that the signal is about 300 times higher than the signal obtained with a constant voltage applied between the plates as in Fig. 5 .

\section{ELECTRIC FIELD IN A PLASMA SHEATH}

We now create, in the measurement chamber, a low density DC argon discharge plasma at low pressure $\mathrm{P}=4.9 \cdot 10^{-5} \mathrm{mbar}$ from a heated filament biased at $-60 \mathrm{~V}$. The vertical profile of the electric field magnitude in the region between the two horizontal plates is obtained by moving both biased plates vertically around the plasma-beam interacting volume. Lock-in detection results from the modulation of the $\mathrm{H}(2 s)$ at $10 \mathrm{~Hz}$. Figure $8(\mathrm{a})$ displays the measured signal at different positions of the beam between the plates for grounded (squares), $-100 \mathrm{~V}$ (circles), $-200 \mathrm{~V}$ (upper triangles), and $-270 \mathrm{~V}$ (lower triangles) biasing of one of the plates while the other one is grounded. We subtract the results for grounded plates from the results with biasing in order to get the part of the signal resulting only from the electric field in the plasma. A signal proportional to

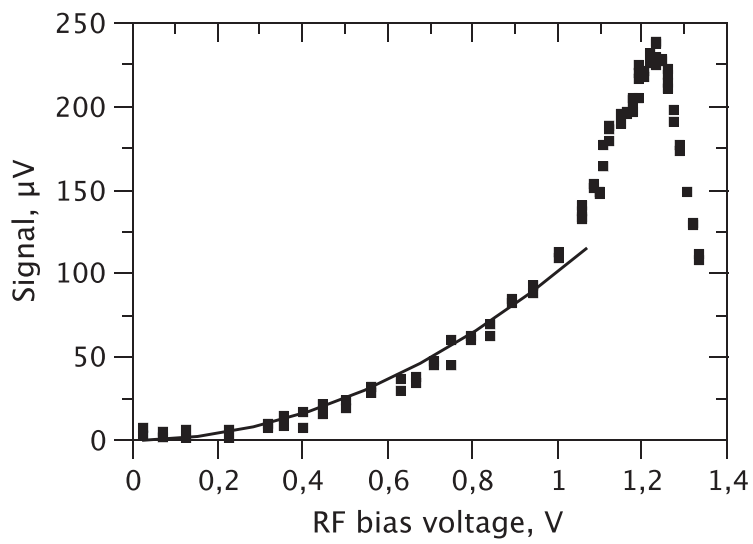

FIG. 7. Lyman- $\alpha$ emission versus radiofrequency potential applied between the plates with an atomic beam; solid line, a parabola fitted to the data; source pressure $P=1.7 \cdot 10^{-5} \mathrm{mbar}$ and discharge current $I_{d}=2.5 \mathrm{~A}$.
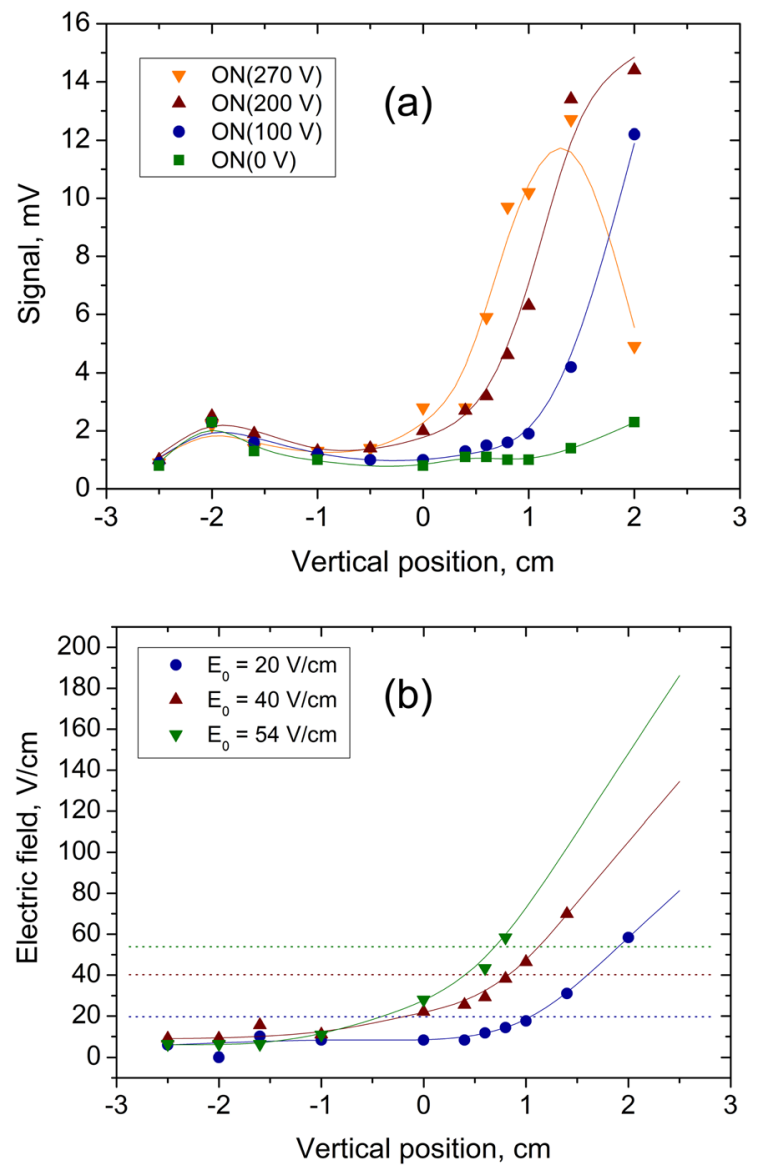

FIG. 8. (a) Lyman- $\alpha$ emission versus position between two parallel plates; (b) Electric field measured in a plasma sheath with a $\mathrm{H}(2 s)$ probe beam; source pressure $P=1.1 \cdot 10^{-4} \mathrm{mbar}$ and discharge current $I_{d}=1.9 \mathrm{~A}$.

the electric field is obtained by taking the square root of this subtraction. Normalization of the electric field is obtained by integration over the distance between the plates and equating this integration to the applied potential. The normalized electric field is plotted in Figure 8(b). We have removed the points corresponding to the region closest to the plate where the probing atomic beam intersects the plate. We clearly observe the Debye shielding in the presence of the plasma: the electric field is much stronger close to the negatively biased electrode due to the formation of a plasma sheath and falls down to a value systematically smaller than the vacuum values (given by the horizontal dotted lines) out of the sheath.

\section{CONCLUSION}

The presented results allowed to recover the thoroughly established underlying science associated to the well-known Lamb shift. The diagnostic uses a fairly current technology for the formation of the $\mathrm{H}(2 s)$ beam. The new method of local non-intrusive measurement of the electric field amplitude that we have presented here therefore relies on a firm foundation. Beside vacuum or plasma as shown in this paper, the investigated medium can also be a diluted fluid. In any case, there is a strong need for such measurements. In the plasma domain, this method could be applied in laboratory experiments, industrial plasmas, ion thrusters, or fusion machines where the knowledge of the local electric field is 
very important and remains a challenging issue. It can be applied as well for constant or fluctuating electric fields. Experiments exploiting the resonant Lamb shift measurements are underway. It is also worth noting that a variety of hydrogenic ions (HeII for example) can then play a double role, as a constituent of the plasma as well as a test particle. Local static or oscillatory magnetic fields could also be measured through the unfolding of the motional $\mathbf{v} \times \mathbf{B}$ field and its effect on the $2 s_{1 / 2}$ lifetime. In conclusion, the results displayed in this letter open completely new tracks for nonintrusive measurements of electric fields.

\section{ACKNOWLEDGMENTS}

The authors dedicate this publication to late Professor R. A. Stern who was the inspirer of this research. The authors are grateful to B. Squizzaro and J.-P. Busso for technical assistance. They acknowledge the help of G. Bachet and G. Prasad for the conception and construction of the experimental set-up. The results presented here were obtained during A. Lejeune's Ph.D. thesis. He benefited from a grant by French Ministère de la Recherche.

${ }^{1}$ A. I. Eriksson et al., Ann. Geophys. 24, 275 (2006).

${ }^{2}$ S. V. Ratinskaia, V. I. Demidov, and K. Rypdal, Phys. Plasmas 9, 4135 (2002).

${ }^{3}$ F. H. Mull, T. P. Crowley, P. M. Schoch, and K. A. Connor, Rev. Sci. Instrum. 70, 959 (1999).
${ }^{4}$ S. Ohshima et al., Rev. Sci. Instrum. 79, $10 F 320$ (2008).

${ }^{5}$ G. Bachet, F. Skiff, F. Doveil, and R. A. Stern, Phys. Plasmas 8, 3535 (2001).

${ }^{6}$ A. Lejeune, G. Bourgeois, and S. Mazouffre, Phys. Plasmas 19, 073501 (2012)

${ }^{7}$ R. A. Stern, private communication (1994).

${ }^{8}$ A. Lejeune, L. Chérigier-Kovacic, and F. Doveil, Appl. Phys. Lett. 99, 181502 (2011).

${ }^{9}$ W. E. Lamb, Jr., Rep. Prog. Phys. 14, 19 (1951).

${ }^{10}$ J. F. Benage, Jr., R. R. Stevens, Jr., and R. A. Stern, Nucl. Instrum. Methods A 274, 13 (1989).

${ }^{11}$ W. E. Lamb, Jr. and R. C. Retherford, Phys. Rev. 72, 241 (1947).

${ }^{12}$ W. E. Lamb, Jr. and R. C. Retherford, Phys. Rev. 79, 549 (1950).

${ }^{13}$ H. A. Bethe and E. E. Salpeter, Quantum Mechanics of One and TwoElectron Atoms (Springer-Verlag, Berlin, 1957).

${ }^{14}$ J. F. Benage, Jr., "Plasma effects on the metastable $\mathrm{H}(2 s)$ atoms," $\mathrm{Ph} . \mathrm{D}$. dissertation (University of Colorado, 1986).

${ }^{15} \mathrm{~A}$. Lejeune, "Mise au point d'un diagnostic optique non intrusif pour la mesure des micro-fluctuations locales des champs électriques dans les plasmas," Thèse de doctorat (Université de Provence, 2010).

${ }^{16}$ G. Prasad, G. Bachet, F. Doveil, L. Chérigier, and R. A. Stern, in Europhysics Conference Abstracts of 1998 ICPP and 25th Conference on Controlled Fusion and Plasma Physics (European Physical Society, Praha, 1998), Vol. 22C, p. 110.

${ }^{17}$ A. Lejeune, L. Chérigier-Kovacic, and F. Doveil, in Europhysics Conference Abstracts 35th Conference on Controlled Fusion and Plasma Physics (European Physical Society, Hersonissos, 2008), Vol. 32D, P1. 186.

${ }^{18}$ M. Bacal et al., Nucl. Instrum. Methods 114, 407 (1974); M. Bacal and W. Reichelt, Rev. Sci. Instrum. 45, 769 (1974).

${ }^{19}$ P. Pradel, F. Roussel, A. S. Schlachter, G. Spiess, and A. Valance, Phys. Rev. A 10, 797 (1974).

${ }^{20}$ D. H. Crandall and D. H. Jaecks, Phys. Rev. A 4, 2271 (1971).

${ }^{21}$ S. R. Lundeen, P. E. Jessop, and F. M. Pipkin, Phys. Rev. Lett. 34, 377 (1975). 